\title{
Analysis of the long-time asymptotic behaviour of the solution of a two-dimensional reaction-diffusion equation
}

\author{
J.N. Ndam \\ Department of Mathematics, University of Jos, P.M.B 2084, Jos, Nigeria \\ Email:ndamj@unijos.edu.ng
}

Copyright (c)2015 J.N. Ndam. This is an open access article distributed under the Creative Commons Attribution License, which permits unrestricted use, distribution, and reproduction in any medium, provided the original work is properly cited.

\begin{abstract}
A reaction-diffusion equation in two dimensions is considered. The long-time asymptotic behaviour of the solution of this equation is examined in terms of uniform diffusion as well as density-dependent diffusion. The results show that in both cases, the solution attains a steady state, but does so more slowly with the variable diffusion coefficient when its magnitude $d<1$.
\end{abstract}

Keywords: Reaction-diffusion equation; Energy function; Poincare inequality; Reflecting boundary conditions.

\section{Introduction}

Mathematical models of diffusion of animal species have been under investigation for a very long time. Okubo and Levin [5], Murray [4], Kot [3], and many more can be consulted on this subject. In particular, the classic Fisher equation for the spread of genes has received a lot of attention by applied mathematicians. This same equation could be used to model the diffusion of animal species with little modifications . It is a nonlinear equation, and so can be very difficult or impossible to solve analytically, hence qualitative methods of analysis of the solutions are often sought. Prominent among such methods is the energy method. A lot of researchers have made use of this procedure to examine the long-time asymptotic behaviour of the solutions of reaction-diffusion equations, including Jones and Sleeman [1] and Lokenath Debnath [2]. They examined the one-dimensional reaction-diffusion equations with constant and density-dependent diffusion coefficients. Thus, we intend to extend the idea to the two-dimensional reaction-diffusion equations with constant and density-dependent diffusion coefficients, which have not been considered. This is necessary because animal species in their natural habitats move on a plain, which is better captured by the two-dimensional equation. 


\section{Main results}

\subsection{The Equation with constant diffusion coefficient}

A reaction diffusion equation in two dimensions is considered. This equation which could be a mathematical model for the dispersal of an animal species in a natural habitat is given by

$\frac{\partial u}{\partial t}=D\left[\frac{\partial^{2} u}{\partial x^{2}}+\frac{\partial^{2} u}{\partial y^{2}}\right]+r u\left(1-\frac{u}{K}\right)-\mu u$

where $D$ is the diffusion coefficient, $r$ is the growth rate of the species, $K$ is the carrying capacity of the environment and $\mu$ is the natural death rate. Using the scalling variables

$$
x^{*}=\sqrt{\frac{r}{D}} x, y^{*}=\sqrt{\frac{r}{D}} y, t^{*}=r t, u^{*}=\frac{u}{K},
$$

we obtain after dropping asterisk, the equation

$\frac{\partial u}{\partial t}=\left[\frac{\partial^{2} u}{\partial x^{2}}+\frac{\partial^{2} u}{\partial y^{2}}\right]+u(1-u)-\phi u$

with $\phi=\frac{\mu}{r}<1$.

We now investigate the asymptotic behaviour of the equation when $t$ is large by using the "Energy method" as explained in the proof of the theorem below. Under the reflecting boundary conditions

$u_{x}(0, y, t)=u_{x}(1, y, t)=0 ; u_{y}(x, 0, t)=u_{y}(x, 1, t)=0$,

the solution of equation (2) attains a steady state for $k>\frac{(1-\phi)^{2}}{4}$ as $t \rightarrow \infty$, where $k$ is a constant.

Proof:

Using the energy method, we define

$$
\begin{gathered}
E(t)=\frac{1}{2} \int_{0}^{1} \int_{0}^{1}\left[u_{x}^{2}+u_{y}^{2}\right] d x d y \\
\frac{d E}{d t}=\int_{0}^{1} \int_{0}^{1}\left[u_{x} u_{x t}+u_{y} u_{y t}\right] d x d y \\
=\int_{0}^{1} \int_{0}^{1}\left\{u_{x}\left[u_{x x}+u_{y y}+f(u)\right]_{x}+u_{y}\left[u_{x x}+u_{y y}+f(u)\right]_{y}\right\} d x d y \\
=\int_{0}^{1} \int_{0}^{1}\left[u_{x} u_{x x x}+u_{x} u_{x y y}+u_{x}^{2} f_{u}+u_{y} u_{x x y}+u_{y} u_{y y y}+u_{y}^{2} f_{u}\right] d x d y \\
=-\int_{0}^{1} \int_{0}^{1}\left(u_{x x}^{2}+u_{y y}^{2}\right) d x d y-2 \int_{0}^{1} \int_{0}^{1} u_{x x} u_{y y} d x d y+\int_{0}^{1} \int_{0}^{1}\left(u_{x}^{2}+u_{y}^{2}\right) f_{u} d x d y .
\end{gathered}
$$

Now, let $m=\max \left|f_{u}\right|$ over all bounded values of $u$, then

$$
\frac{d E}{d t} \leq-\int_{0}^{1} \int_{0}^{1}\left(u_{x x}+u_{y y}\right)^{2} d x d y+2 m E(t)
$$

Using the Poincare inequality

$$
\int_{0}^{1} u_{x x}^{2} d x \geq k \int_{0}^{1} u_{x}^{2} d x
$$

in one dimension, one obtains the inequality

$$
\int_{0}^{1} \int_{0}^{1}\left(u_{x x}+u_{y y}\right)^{2} d x d y \geq k \int_{0}^{1} \int_{0}^{1}\left(u_{x}^{2}+u_{y}^{2}\right) d x d y,
$$

hence

$$
\begin{gathered}
\frac{d E}{d t} \leq-k \int_{0}^{1} \int_{0}^{1}\left(u_{x}^{2}+u_{y}^{2}\right) d x d y+2 m E(t)=-2 k E(t)+2 m E(t) . \\
E(t)=E(0) e^{2(m-k) t} .
\end{gathered}
$$

But from equation $(2), f(u)=u(1-u)-\phi u$, hence $m=\max \left|f_{u}\right|=\frac{(1-\phi)^{2}}{4}$. Therefore, $u$ attains a steady-state when $k>\frac{(1-\phi)^{2}}{4}$, as $t \rightarrow \infty$. 


\subsection{The equation with density-dependent diffusion}

In this section,we investigate the long-time asymptotic behaviour of the equation

$u_{t}=\left[D(u) u_{x}\right]_{x}+\left[D(u) u_{y}\right]_{y}+u(1-u)-\phi u$

where $D(u)$ is the dimensionless density-dependent diffusion coefficient. Using the same energy function defined above, one obtains

$$
\begin{aligned}
\frac{d E}{d t} & =\int_{0}^{1} \int_{0}^{1} u_{x}\left[\left(D(u) u_{x}\right)_{x}+\left(D(u) u_{y}\right)_{y}+f(u)\right]_{x} d x d y \\
& +\int_{0}^{1} \int_{0}^{1} u_{y}\left[\left(D(u) u_{x}\right)_{x}+\left(D(u) u_{y}\right)_{y}+f(u)\right]_{y} d x d y
\end{aligned}
$$

where $f(u)=u(1-u)-\phi u$.

$$
\begin{array}{r}
\frac{d E}{d t}=\int_{0}^{1} \int_{0}^{1} u_{x}\left[\left(D(u) u_{x}\right)_{x}+\left(D(u) u_{y}\right)_{y}\right]_{x} d x d y+\int_{0}^{1} \int_{0}^{1} u_{y}\left[\left(D(u) u_{x}\right)_{x}+\left(D(u) u_{y}\right)_{y}\right]_{y} d x d y \\
+\int_{0}^{1} \int_{0}^{1}\left(u_{x}^{2}+u_{y}^{2}\right) f_{u} d x d y
\end{array}
$$

Evaluating the first two integrals by parts, one obtains

$$
\begin{array}{r}
\frac{d E}{d t}=-\int_{0}^{1} \int_{0}^{1} u_{x x}\left[D_{u}(u)\left(u_{x}^{2}+u_{y}^{2}\right)+D(u)\left(u_{x x}+u_{y y}\right)\right] d x d y \\
-\int_{0}^{1} \int_{0}^{1} u_{y y}\left[D_{u}(u)\left(u_{x}^{2}+u_{y}^{2}\right)+D(u)\left(u_{x x}+u_{y y}\right)\right] d x d y+\int_{0}^{1} \int_{0}^{1}\left(u_{x}^{2}+u_{y}^{2}\right) f_{u} d x d y
\end{array}
$$

Hence we have

$$
\begin{aligned}
& \frac{d E}{d t} \leq-\int_{0}^{1} \int_{0}^{1}\left[\left(u_{x x}+u_{y y}\right)\left(u_{x}^{2}+u_{y}^{2}\right) D_{u}(u)+D(u)\left(u_{x x}+u_{y y}\right)^{2}\right] d x d y+2 m E(t) \\
& \frac{d E}{d t} \leq-\int_{0}^{1} \int_{0}^{1} d\left(u_{x x}+u_{y y}\right)^{2} d x d y+2 m E(t)
\end{aligned}
$$

where $d=\max |D(u)|$ and noting that $D_{u}(u)=0$ at the maximum point.

$$
\begin{array}{r}
\frac{d E}{d t} \leq-k d \int_{0}^{1} \int_{0}^{1}\left(u_{x}^{2}+u_{y}^{2}\right) d x d y+2 m E(t) \leq-2 k d E(t)+2 m E(t) \\
\frac{d E}{d t} \leq-2(m-k d) E(t) \Rightarrow E(t) \leq E(0) e^{2(m-k d) t} .
\end{array}
$$

Hence the solution of (4) attains a steady state for $k>\frac{m}{d}=\frac{(1-\phi)^{2}}{4 d}$, as $t \rightarrow \infty$.

\section{Conclusion}

Two cases of reaction-diffusion equations are considered, one with constant diffusion coefficient and another with density-dependent diffusion coefficient. In both cases, criteria for the attainment of uniform state for the solutions were obtained. The solution of the density-dependent reaction-diffusion equation attains a steady state slower than that with constant diffusion coefficient when $d<1$.

\section{Acknowledgement}

I thank my employer, the University of Jos, and the Department of Mathematics, for the privilege to use some of their facilities in the course of this research work. 


\section{References}

[1] D.S. Jones and B.D. Sleeman, Differential Equations and Mathematical Biology, Chapman and Hall/CRC, USA, 2003.

[2] L. Debnath, Nonlinear Partial Differential Equations for Scientists and Engineers, Springer Science+Business Media, New York, 2012.

[3] M. Kot, Elements of Mathematical Biology, Cambridge University Press, United Kingdom, 2001.

[4] J.D. Murray, Mathematical Biology I. An Introduction, Springer-Verlag, Berlin, 2002.

[5] A. Okubo and S.A. Levin, Diffusion and Ecological Problems: Modern Perspectives, Springer, New York, 2001. 